Pacific

Journal of

Mathematics

ELLIPTIC SURFACES AND AMPLE VECTOR BUNDLES

Antonio Lanteri And Hidetoshi Maeda 


\title{
ELLIPTIC SURFACES AND AMPLE VECTOR BUNDLES
}

\author{
Antonio Lanteri and Hidetoshi Maeda
}

Let $\mathcal{E}$ be an ample vector bundle of rank $n-2 \geq 2$ on a complex projective manifold $X$ of dimension $n$ having a section whose zero locus is a smooth surface $Z$. We determine the structure of pairs $(X, \mathcal{E})$ as above under the assumption that $Z$ is a properly elliptic surface. This generalizes known results on threefolds containing an elliptic surface as a smooth ample divisor. Among the applications we prove a conjecture relating the Kodaira dimension of $X$ to that of $Z$, and we show that if $0 \leq \kappa(Z) \leq 1$, then $p_{g}(Z)>0$ unless $X$ is a $\mathbb{P}^{n-2}$-bundle over a smooth surface $S$ with $p_{g}(S)=0$.

\section{Introduction.}

Consider the following set-up.

(*) $X$ is a smooth complex projective variety of dimension $n$ and $\mathcal{E}$ is an ample vector bundle of rank $n-2 \geq 2$ on $X$ such that there exists a section $s \in \Gamma(\mathcal{E})$ whose zero locus $Z:=(s)_{0}$ is a smooth surface.

According to a general philosophy coming from the study of ample divisors [S1], if $Z$ is special, then $X$ has to be special as well. By relying on the study of the nefness of the adjoint bundle $K_{X}+\operatorname{det} \mathcal{E}[\mathbf{M}]$, the structure of pairs $(X, \mathcal{E})$ as above is now well understood when $\kappa(Z) \leq 0$ in view of $[\mathbf{L M 1}$ and $[\mathbf{L} 1]$. In this paper we investigate the structure of $(X, \mathcal{E})$ when $Z$ is a properly elliptic surface.

Our result is as follows.

Theorem. Let $X, \mathcal{E}$ and $Z$ be as in (*) and assume further that $Z$ is an elliptic surface with $\kappa(Z)=1$. Then one of the following conditions holds.

(a) $X=\mathbb{P}_{S}(\mathcal{F})$, where $\mathcal{F}$ is an ample vector bundle of rank $n-1$ over a smooth surface $S, \mathcal{E}=\pi^{*} \mathcal{V} \otimes H$, where $H=H(\mathcal{F})$ is the tautological line bundle on $X, \mathcal{V}$ is a vector bundle of rank $n-2$ on $S, \pi: X \rightarrow S$ is the bundle projection and its restriction to $Z, \pi_{\mid Z}: Z \rightarrow S$ is a birational morphism, but not an isomorphism.

(b) There exist a birational morphism $f: X \rightarrow X^{\prime}$ expressing $X$ as a projective manifold $X^{\prime}$ blown-up at a finite set $B$ of points (possibly empty) and an ample vector bundle $\mathcal{E}^{\prime}$ of rank $n-2$ on $X^{\prime}$ such that $\mathcal{E}=f^{*} \mathcal{E}^{\prime} \otimes\left[-f^{-1}(B)\right]$ and that $K_{X^{\prime}}+\operatorname{det} \mathcal{E}^{\prime}$ is nef. The triplet 
$\left(X^{\prime}, \mathcal{E}^{\prime}, Z^{\prime}:=f(Z)\right)$ satisfies $(*)$ and $\kappa\left(Z^{\prime}\right)=1$. Moreover $X^{\prime}$ is endowed with a morphism $\varphi: X^{\prime} \rightarrow Y$ onto a smooth curve $Y$ of genus $g(Y)=h^{1,0}(Z)$, whose general fibre $F$ is a projective manifold of dimension $n-1$ satisfying the condition $K_{F}+\operatorname{det} \mathcal{E}_{F}^{\prime}=\mathcal{O}_{F} ; \varphi$ induces on $Z^{\prime}$ the elliptic fibration, and $\varphi_{\mid Z^{\prime}}: Z^{\prime} \rightarrow Y$ has no multiple fibres.

Pairs $\left(F, \mathcal{E}_{F}^{\prime}\right)$ of this kind have been classified in [PSW].

In the 80 's Sommese investigated the structure of projective threefolds containing an elliptic surface as a hyperplane section, or more generally as an ample divisor [S2, Theorem 3.1]. Our Theorem can be viewed as a complete generalization of what Sommese and Shepherd-Barron proved in that setting (see [D, Theorem 0.7]). In the threefold case Sommese proved that the elliptic fibration cannot have multiple fibres [S2, Claim 3.1.4] (see also [S3, Lemma 0.5.1]), by relying on a formula he obtained for the plurigenera of the surface. Our proof of the fact that $\varphi_{\mid Z^{\prime}}: Z^{\prime} \rightarrow Y$ has no multiple fibres is conceptually much easier and we derive from this fact a formula expressing both $h^{0}\left(m\left(K_{X^{\prime}}+\operatorname{det} \mathcal{E}^{\prime}\right)\right)$ and the $m$-th genus $P_{m}(Z)$ for $m>0$ as a linear polynomial in $m$ (Corollary (1.3)). Moreover we show that in case (b) the surface $Z$ must have positive geometric genus, which was not explicitly noted even in the setting of ample divisors (Corollary (2.2)). In particular, if $p_{g}(Z)=0$ then $(X, \mathcal{E})$ is as in case (a). We show that this holds also when $\kappa(Z)=0$, which gives some more evidence for a conjecture stated in $[\mathbf{L 2}]$. As a consequence of our Theorem we also see that a product of an elliptic curve with a smooth curve of positive genus can never occur as the zero locus of a section of an ample vector bundle (Corollary (1.4)).

As another application of our Theorem we prove that if $X, \mathcal{E}$ and $Z$ are as in $(*)$ and $\kappa(Z)<\operatorname{dim} Z$, then $\kappa(X)=-\infty$ (Corollary (2.1)). This fact, which was conjectured in [LM1], also comes from a recent, more general, theory of normal pairs with $\mathbb{Q}$-effective normal sheaf, developed by Peternell, Schneider and Sommese [PSS].

The present paper was prepared in the framework of the National Research Project "Algebraic Geometry, Commutative Algebra and Computational Aspects", supported by the M.U.R.S.T. of the Italian Government. The second author was also partially supported by Grant-in-Aid for Encouragement of Young Scientists (No. 11740027), the Ministry of Education, Science, Sports and Culture, Japan, as well as Waseda University Grant for Special Research Projects (No. 99A-192). Both authors would like to thank the M.U.R.S.T. and the University of Milan for making this collaboration possible.

\section{Preliminaries.}

In this paper we will work over the complex number field $\mathbb{C}$. We use the standard notation from algebraic geometry. We make no distinction between 
vector bundles and locally free sheaves. The tensor products of line bundles are denoted additively, while we use multiplicative notation for intersection products in Chow rings. The pull-back $i^{*} \mathcal{E}$ of a vector bundle $\mathcal{E}$ on $X$ by an embedding $i: Y \hookrightarrow X$ is denoted by $\mathcal{E}_{Y}$. If $X$ is a smooth projective variety, the canonical bundle of $X$ is denoted by $K_{X}$ and $\kappa(X)$ stands for the Kodaira dimension of $X$. For any $m \geq 1$ the $m$-th plurigenus $h^{0}\left(m K_{X}\right)$ is denoted by $P_{m}(X)$. In particular, for $m=1$, we denote by $p_{g}(X)=P_{1}(X)$ the geometric genus of $X$. Moreover, if $X$ is a smooth projective curve, the genus of $X$ is denoted by $g(X)$ instead of $p_{g}(X)$.

Let $X, \mathcal{E}$ and $Z$ be as in (*). Since $Z$ is smooth of the expected dimension, we have $N_{Z / X} \cong \mathcal{E}_{Z}$, where $N_{Z / X}$ denotes the normal bundle of $Z$ in $X$. Hence, by adjunction, we get

$$
K_{Z}=\left(K_{X}\right)_{Z}+\operatorname{det} N_{Z / X}=\left(K_{X}+\operatorname{det} \mathcal{E}\right)_{Z} .
$$

We will use this fact over and over. The following fact follows easily from $[\mathrm{M}$, Theorem].

Lemma 0.1. Let $X, \mathcal{E}$ and $Z$ be as in $(*)$ and assume that $\kappa(Z) \geq 0$. Then either $K_{X}+\operatorname{det} \mathcal{E}$ is nef, or one of the following cases occurs:

(i) $X$ contains an effective divisor $E$ such that

$$
\left(E, \mathcal{O}_{E}(E), \mathcal{E}_{E}\right) \cong\left(\mathbb{P}^{n-1}, \mathcal{O}_{\mathbb{P}}(-1), \mathcal{O}_{\mathbb{P}}(1)^{\oplus(n-2)}\right) ;
$$

(ii) $X=\mathbb{P}_{S}(\mathcal{F})$ for some ample vector bundle $\mathcal{F}$ of rank $n-1$ on a smooth surface $S$ and $\mathcal{E}=\pi^{*} \mathcal{V} \otimes H$ for some vector bundle $\mathcal{V}$ of rank $n-2$ on $S$, where $H$ is the tautological line bundle on $X$ and $\pi: X \rightarrow S$ is the bundle projection.

We recall the following fact.

Lemma 0.2 ([LM1, Lemma 5.1]). Let $\mathcal{E}$ be an ample vector bundle of rank $r \geq 2$ on a smooth projective variety $X$ of dimension $n \geq 2$ and assume that $X$ contains a smooth divisor $E$ such that

$$
\left(E, \mathcal{O}_{E}(E), \mathcal{E}_{E}\right) \cong\left(\mathbb{P}^{n-1}, \mathcal{O}_{\mathbb{P}}(-1), \mathcal{O}_{\mathbb{P}}(1)^{\oplus r}\right) .
$$

Let $f: X \rightarrow X^{\prime}$ be the blow-down of $E$ to a point $x \in X^{\prime}$. Then there exists an ample vector bundle $\mathcal{E}^{\prime}$ of rank $r$ on $X^{\prime}$ such that

$$
\mathcal{E}=f^{*} \mathcal{E}^{\prime} \otimes \mathcal{O}_{X}(-E) .
$$

In connection with this we prove the following lemma, inspired by $[\mathbf{L} 1$, Lemma 2.2].

Lemma 0.3. Let $(X, \mathcal{E}), E, f, x$ and $\left(X^{\prime}, \mathcal{E}^{\prime}\right)$ be as above. Then $X^{\prime}$ cannot contain any submanifold $F$ of positive dimension, such that $x \in F$ and

$$
\left(F, \mathcal{E}_{F}^{\prime}\right) \cong\left(\mathbb{P}^{s}, \mathcal{O}_{\mathbb{P}}(1)^{\oplus r}\right) .
$$


Proof. By contradiction, assume that $X^{\prime}$ contains such an $F$, and let $\widetilde{F}$ be the proper transform of $F$ via $f$. Note that $\widetilde{F}$ is a $\mathbb{P}^{1}$-bundle over $\mathbb{P}\left(N_{x / F}^{\vee}\right)=\mathbb{P}^{s-1}$, whose fibres are the proper transforms of the lines on $F$ passing through $x$. Moreover $E \cap \widetilde{F}$ is a section of this $\mathbb{P}^{1}$-bundle. So, if $l$ is a fibre of $\widetilde{F}$, then $\mathcal{O}_{X}(E) l=\mathcal{O}_{\widetilde{F}}(E) l=1$. Since $\mathcal{E}_{F}^{\prime} \cong \mathcal{O}_{\mathbb{P}}(1)^{\oplus r}$ we thus get from $(0.2 .1)$

$$
\mathcal{E}_{l}=\left(f^{*} \mathcal{E}^{\prime} \otimes \mathcal{O}_{X}(-E)\right)_{l}=\mathcal{O}_{l}^{\oplus r},
$$

contradicting the ampleness of $\mathcal{E}$.

Remark 0.4. In particular Lemma 0.3 shows that for the pair $\left(X^{\prime}, \mathcal{E}^{\prime}\right)$ obtained after the contraction $f$ above,

(1) $X^{\prime}$ cannot be a $\mathbb{P}^{s}$-bundle, $s \geq 1$, over a smooth projective variety $W, \operatorname{dim} W \geq 0$, with $\mathcal{E}_{F}^{\prime}=\mathcal{O}_{\mathbb{P}}(1)^{\oplus r}$ for any fibre $F$ of the projection $X^{\prime} \rightarrow W$, and

(2) if $X^{\prime}$ contains an exceptional divisor $E^{\prime}$ such that

$$
\left(E^{\prime}, \mathcal{O}_{E^{\prime}}\left(E^{\prime}\right), \mathcal{E}_{E^{\prime}}^{\prime}\right) \cong\left(\mathbb{P}^{n-1}, \mathcal{O}_{\mathbb{P}}(-1), \mathcal{O}_{\mathbb{P}}(1)^{\oplus r}\right)
$$

then $E$ and $f^{-1}\left(E^{\prime}\right)$ are two disjoint exceptional divisors in $X$.

The following lemma will be useful in Section 1 to add something more in case (b) of our Theorem.

Lemma 0.5. Let $Z$ be a minimal elliptic surface with $\kappa(Z)=1$, and let $\psi: Z \rightarrow Y$ denote the elliptic fibration over a smooth projective curve $Y$. If $\psi$ has no multiple fibres and if $K_{Z}=\psi^{*} M$ for some ample line bundle $M$ on $Y$, then the $m$-th plurigenus of $Z$ is given by the formula

(0.5.1) $\quad P_{m}(Z)=m\left(\chi\left(\mathcal{O}_{Z}\right)+2 g(Y)-2\right)+1-g(Y)$ for every $m \geq 2$.

Moreover, (0.5.1) is valid even for $m=1$, unless $M=K_{Y}$.

Proof. Assume first that $m \geq 2$. We note that $\chi\left(m K_{Z}\right)=\chi\left(\mathcal{O}_{Z}\right)$, because $K_{Z}^{2}=0$. Moreover, by Serre duality we have $h^{2}\left(m K_{Z}\right)=h^{0}\left(-(m-1) K_{Z}\right)$. On the other hand, $K_{Z} A>0$ for any ample line bundle $A$ on $Z$, since $\kappa(Z)=1$. So $h^{2}\left(m K_{Z}\right)=0$. Consequently

$$
\begin{aligned}
P_{m}(Z) & =h^{0}\left(m K_{Z}\right)-h^{1}\left(m K_{Z}\right)+h^{2}\left(m K_{Z}\right)+h^{1}\left(m K_{Z}\right)-h^{2}\left(m K_{Z}\right) \\
& =\chi\left(m K_{Z}\right)+h^{1}\left(m K_{Z}\right)-h^{2}\left(m K_{Z}\right) \\
& =\chi\left(\mathcal{O}_{Z}\right)+h^{1}\left(m K_{Z}\right) .
\end{aligned}
$$

From now on, let us calculate $h^{1}\left(m K_{Z}\right)$. Since $\psi$ has no multiple fibres, the canonical bundle formula for elliptic fibrations $[\mathbf{F}$, Theorem 15, p. 176] tells us that $K_{Z}=\psi^{*}\left(K_{Y}+L\right)$, where $L$ is the dual of the line bundle $R^{1} \psi_{*} \mathcal{O}_{Z}$. We note that $\operatorname{deg} L=\chi\left(\mathcal{O}_{Z}\right) \geq 0$ [F, Lemmas 13 and 14, p. 176]. Furthermore, since $\psi_{*} \mathcal{O}_{Z}=\mathcal{O}_{Y}$, the homomorphism $\psi^{*}: \operatorname{Pic}(Y) \rightarrow \operatorname{Pic}(Z)$ 
is injective. Therefore, by assumption, $M=K_{Y}+L$. Now we consider the exact sequence

$$
0 \rightarrow H^{1}\left(\psi_{*}\left(m K_{Z}\right)\right) \rightarrow H^{1}\left(m K_{Z}\right) \rightarrow H^{0}\left(R^{1} \psi_{*}\left(m K_{Z}\right)\right) \rightarrow 0
$$

induced by the Leray spectral sequence

$$
E_{2}^{p, q}=H^{p}\left(R^{q} \psi_{*}\left(m K_{Z}\right)\right) \Longrightarrow E^{p+q}=H^{p+q}\left(m K_{Z}\right) .
$$

This gives

$$
h^{1}\left(m K_{Z}\right)=h^{1}\left(\psi_{*}\left(m K_{Z}\right)\right)+h^{0}\left(R^{1} \psi_{*}\left(m K_{Z}\right)\right) .
$$

As to the first summand, by the projection formula, we see that $\psi_{*}\left(m K_{Z}\right)=$ $\psi_{*} \psi^{*}(m M)=m M$. Moreover, we can write $m M=K_{Y}+(m-1) M+L$. Since $\operatorname{deg}((m-1) M+L)>0$, we get $h^{1}\left(\psi_{*}\left(m K_{Z}\right)\right)=h^{1}(m M)=0$. So it suffices to calculate $h^{0}\left(R^{1} \psi_{*}\left(m K_{Z}\right)\right)$. From the projection formula, we obtain

$$
\begin{aligned}
R^{1} \psi_{*}\left(m K_{Z}\right) & =R^{1} \psi_{*} \psi^{*}(m M)=R^{1} \psi_{*} \mathcal{O}_{Z} \otimes m M \\
& =m M-L=K_{Y}+(m-1) M .
\end{aligned}
$$

Since $\operatorname{deg}((m-1) M)>0$, the Riemann-Roch theorem for $K_{Y}+(m-1) M$ gives

$$
\begin{aligned}
h^{0}\left(R^{1} \psi_{*}\left(m K_{Z}\right)\right) & =h^{0}\left(K_{Y}+(m-1) M\right) \\
& =2 g(Y)-2+(m-1) \operatorname{deg} M+1-g(Y) \\
& =2 g(Y)-2+(m-1)\left(2 g(Y)-2+\chi\left(\mathcal{O}_{Z}\right)\right)+1-g(Y) .
\end{aligned}
$$

Combining (0.5.2) with (0.5.3), we get the desired formula.

When $m=1$, an easy calculation shows that the right-hand side of (0.5.1) is $P_{1}(Z)+g(Y)-h^{1}\left(\mathcal{O}_{Z}\right)$. If $M \neq K_{Y}$, i.e., $L$ is not trivial, then it follows from $\left[\mathbf{F}\right.$, Lemma 14, p. 176] that $g(Y)=h^{1}\left(\mathcal{O}_{Z}\right)$. Therefore $(0.5 .1)$ is still true unless $M=K_{Y}$.

\section{The structure of $(X, \mathcal{E})$.}

1.1. Proof of the Theorem. Assume first that $K_{X}+\operatorname{det} \mathcal{E}$ is not nef. Then, since $\kappa(Z)=1,(X, \mathcal{E})$ is either of type (i) or of type (ii) in Lemma 0.1.

Assume that $(X, \mathcal{E})$ is of type (ii). Let $s_{F}$ denote the restriction of $s$ to any fibre $F$ of $\pi$. Then, since $s_{F} \in \Gamma\left(\mathcal{O}_{\mathbb{P}^{n-2}}(1)^{\oplus(n-2)}\right), Z \cap F=\left(s_{F}\right)_{0}$ is a linear subspace of dimension $\geq 0$ in $F$. Hence the restriction $\pi_{\mid Z}: Z \rightarrow S$ of $\pi$ to $Z$ is surjective. We thus conclude that $\pi_{\mid Z}$ is birational. However, if $\pi_{\mid Z}$ is an isomorphism, then $\operatorname{Pic}(X) \cong \operatorname{Pic}(Z) \times \mathbb{Z}$, which contradicts the fact that the restriction homomorphism $\operatorname{Pic}(X) \rightarrow \operatorname{Pic}(Z)$ is injective [LM2, (1.1.6)]. Thus we are in case (a) of the Theorem. 
Now assume that $(X, \mathcal{E})$ is of type (i). Set $l=Z \cap E$. Then the same argument as that in the proof of [LM1, Corollary 5.2] shows that $l$ is a (-1)curve in $Z$. Let $f_{1}: X \rightarrow X_{1}$ be the blow-down of $E$. Then $Z_{1}:=f_{1}(Z)$ is a smooth projective surface in $X_{1}$ because $f_{1 \mid Z}: Z \rightarrow Z_{1}$ is nothing but the contraction of $l$. In particular $\kappa\left(Z_{1}\right)=1$. Moreover, by Lemma 0.2 there exists an ample vector bundle $\mathcal{E}_{1}$ of rank $n-2$ on $X_{1}$ such that $\mathcal{E}=$ $f_{1}^{*} \mathcal{E}_{1} \otimes \mathcal{O}_{X}(-E)$. If we let $s_{1} \in \Gamma\left(\mathcal{E}_{1}\right)$ denote the section corresponding to $s$, then $\left(s_{1}\right)_{0}=Z_{1}$, so that $X_{1}, \mathcal{E}_{1}$ and $Z_{1}$ also satisfy condition $(*)$ in the Introduction. However, note that $\left(X_{1}, \mathcal{E}_{1}\right)$ is never of type (ii) by virtue of Remark 0.4 (1). Therefore $\left(X_{1}, \mathcal{E}_{1}\right)$ must be of type (i), if $K_{X_{1}}+\operatorname{det} \mathcal{E}_{1}$ is not nef. For this reason, when $K_{X_{1}}+\operatorname{det} \mathcal{E}_{1}$ is not yet nef, we can apply the same argument as above to $X_{1}, \mathcal{E}_{1}$ and $Z_{1}$, and continue in this manner. It should be emphasized that if $X_{1}$ contains $E_{1}$ such that

$$
\left(E_{1}, \mathcal{O}_{E_{1}}\left(E_{1}\right), \mathcal{E}_{1 E_{1}}\right) \cong\left(\mathbb{P}^{n-1}, \mathcal{O}_{\mathbb{P}}(-1), \mathcal{O}_{\mathbb{P}}(1)^{\oplus(n-2)}\right),
$$

then $E \cap f_{1}^{-1}\left(E_{1}\right)=\emptyset$ by Remark 0.4 (2), so that we can contract $E$ and $f_{1}^{-1}\left(E_{1}\right)$ at the same time. This procedure must come to an end after a finite number of repetitions. Therefore we conclude that there exist a birational morphism $f: X \rightarrow X^{\prime}$ expressing $X$ as a projective manifold $X^{\prime}$ blown-up at a finite set $B$ of points and an ample vector bundle $\mathcal{E}^{\prime}$ of rank $n-2$ on $X^{\prime}$ satisfying condition $(*)$, such that $\mathcal{E}=f^{*} \mathcal{E}^{\prime} \otimes\left[-f^{-1}(B)\right]$ and that $K_{X^{\prime}}+\operatorname{det} \mathcal{E}^{\prime}$ is nef unless $(X, \mathcal{E})$ is as in case (a) of the Theorem.

From this, in order to complete our analysis, it suffices to investigate the structure of $(X, \mathcal{E})$ when the adjoint bundle $K_{X}+\operatorname{det} \mathcal{E}$ is nef. So in what follows, we assume that $K_{X}+\operatorname{det} \mathcal{E}$ is nef. Then, by [KMM, Theorem 3-2-1] there exists a morphism $\varphi: X \rightarrow Y$ with connected fibres from $X$ onto a normal projective variety $Y$ such that $K_{X}+\operatorname{det} \mathcal{E}=\varphi^{*} M$ for some ample line bundle $M$ on $Y$.

If $\operatorname{dim} Y=0$, then $K_{X}+\operatorname{det} \mathcal{E}=\mathcal{O}_{X}$. Recalling that $K_{Z}=\left(K_{X}+\operatorname{det} \mathcal{E}\right)_{Z}$, we have $K_{Z}=\mathcal{O}_{Z}$, which contradicts our assumption on $\kappa(Z)$. Hence $\operatorname{dim} Y \geq 1$. Moreover, since $K_{X}+\operatorname{det} \mathcal{E}$ is nef in the present case, $K_{Z}$ is nef, i.e., $Z$ is a minimal surface with $\kappa(Z)=1$. In particular we have $K_{Z}^{2}=0$.

We first claim that $\operatorname{dim} Y=1$. To see this, suppose to the contrary that $\operatorname{dim} Y \geq 2$. Then we can find effective divisors $D_{1}, D_{2} \in\left|m\left(K_{X}+\operatorname{det} \mathcal{E}\right)\right|$ for some $m>0$ such that every irreducible component of $D_{1} \cap D_{2}$ has dimension $n-2$. Applying [BG, Theorem 2.5] to $\mathcal{E}, D_{1}$ and $D_{2}$, we have

$$
m^{2} K_{Z}^{2}=m^{2}\left(K_{X}+\operatorname{det} \mathcal{E}\right)^{2} Z=\left(D_{1} \cap D_{2}\right) c_{n-2}(\mathcal{E})>0,
$$

which gives a contradiction. Hence $\operatorname{dim} Y=1$. As a direct consequence of this, $Y$ is a smooth curve and $\varphi$ is flat. Take a general fibre $F$ of $\varphi$. Then, since $K_{F}+\operatorname{det} \mathcal{E}_{F}=\mathcal{O}_{F}, F$ is a Fano manifold of dimension $n-1$ and $\mathcal{E}_{F}$ is an ample vector bundle of rank $n-2$ on $F$. 
Next we claim that $\varphi_{\mid Z}: Z \rightarrow Y$ is the elliptic fibration. Indeed, $\varphi_{\mid Z}$ is clearly surjective, and satisfies $K_{Z}=\varphi_{\mid Z}^{*} M$. Set $f:=Z \cap F$ for a general fibre $F$ of $\varphi$. Then $f$ is a 1-equidimensional smooth fibre of $\varphi_{\mid Z}$. Moreover, $f$ is the zero locus of the section $s_{F} \in \Gamma\left(\mathcal{E}_{F}\right)$. Therefore $H^{0}(f, \mathbb{Z}) \cong H^{0}(F, \mathbb{Z})=\mathbb{Z}$ by $[\mathbf{L M 2},(1.1 .1)]$ (note that its proof is valid without assuming the connectedness of $f$ ). So $f$ is a smooth curve in $Z$. Combining this with the fact that $K_{Z}=\varphi_{\mid Z}^{*} M$, we see that $f$ is an elliptic curve. Since $\kappa(Z)=1, Z$ admits a unique elliptic fibration, and so the result is proved.

Next we claim that $Y$ has genus

$$
g(Y)=h^{1}\left(\mathcal{O}_{Z}\right) .
$$

To see this, take a general fibre $F$ of $\varphi$. Then $h^{i}\left(\mathcal{O}_{F}\right)=0$ for all $i>0$, because $F$ is Fano. Hence $R^{i} \varphi_{*} \mathcal{O}_{X}=0$ for all $i>0$. Since $\varphi_{*} \mathcal{O}_{X}=\mathcal{O}_{Y}$, we have

$$
H^{1}\left(\mathcal{O}_{X}\right)=H^{1}\left(\varphi_{*} \mathcal{O}_{X}\right)=H^{1}\left(\mathcal{O}_{Y}\right)
$$

On the other hand, it follows from $[\mathbf{L M} 2,(1.1 .3)]$ that $H^{1}\left(\mathcal{O}_{X}\right) \cong H^{1}\left(\mathcal{O}_{Z}\right)$. Thus $h^{1}\left(\mathcal{O}_{Y}\right)=h^{1}\left(\mathcal{O}_{Z}\right)$, as required.

Finally we show that $\varphi_{\mid Z}$ has no multiple fibres. First note that $m\left(K_{X}+\right.$ $\operatorname{det} \mathcal{E})=\varphi^{*}(m M)$ for every $m \geq 1$. As we have seen, $R^{i} \varphi_{*} \mathcal{O}_{X}=0$ for all $i>$ 0 . Combining this with the projection formula, we have $R^{i} \varphi_{*} \varphi^{*}(m M)=0$ for all $i>0$. Moreover, since $\varphi_{*} \mathcal{O}_{X}=\mathcal{O}_{Y}$, the projection formula also tells us that $\varphi_{*} \varphi^{*}(m M)=m M$. Therefore

$$
h^{0}\left(m\left(K_{X}+\operatorname{det} \mathcal{E}\right)\right)=h^{0}\left(\varphi^{*}(m M)\right)=h^{0}(m M) \quad \text { for every } \quad m \geq 1 .
$$

Furthermore, by the Kodaira vanishing theorem,

$$
0=h^{1}\left(m\left(K_{X}+\operatorname{det} \mathcal{E}\right)\right)=h^{1}(m M) \quad \text { for each } m \geq 1,
$$

since $m\left(K_{X}+\operatorname{det} \mathcal{E}\right)=K_{X}+\left((m-1)\left(K_{X}+\operatorname{det} \mathcal{E}\right)+\operatorname{det} \mathcal{E}\right)$ and $K_{X}+\operatorname{det} \mathcal{E}$ is nef. In particular, from (1.1.3) we get

$$
h^{0}(m M)=m \operatorname{deg} M+1-g(Y) \quad \text { for every } \quad m \geq 1 .
$$

Now we consider the exact sequence

$$
0 \rightarrow m\left(K_{X}+\operatorname{det} \mathcal{E}\right) \otimes \mathcal{I}_{Z} \rightarrow m\left(K_{X}+\operatorname{det} \mathcal{E}\right) \rightarrow m K_{Z} \rightarrow 0,
$$

where $\mathcal{I}_{Z}$ is the ideal sheaf of $Z$. We know that $\varphi_{\mid Z}$ is surjective. In other words, $Z$ is not contained in a finite union of fibres of $\varphi$. This implies that $h^{0}\left(m\left(K_{X}+\operatorname{det} \mathcal{E}\right) \otimes \mathcal{I}_{Z}\right)=0$ for every $m \geq 1$, because $m\left(K_{X}+\operatorname{det} \mathcal{E}\right)=$ $\varphi^{*}(m M)$ and every global section of $m\left(K_{X}+\operatorname{det} \mathcal{E}\right)$ is the pullback of a global section of $m M$ by (1.1.2). Thus we have from the exact sequence above

$$
h^{0}\left(m\left(K_{X}+\operatorname{det} \mathcal{E}\right)\right) \leq h^{0}\left(m K_{Z}\right) \quad \text { for all } \quad m \geq 1
$$


In particular, combining (1.1.2) with (1.1.5) for $m=1$, we get

$$
h^{0}(M) \leq h^{0}\left(K_{Z}\right)\left(=h^{2}\left(\mathcal{O}_{Z}\right)\right) .
$$

Hence, by recalling (1.1.1),

$h^{0}(M) \leq h^{2}\left(\mathcal{O}_{Z}\right)-h^{1}\left(\mathcal{O}_{Z}\right)+h^{0}\left(\mathcal{O}_{Z}\right)+h^{1}\left(\mathcal{O}_{Z}\right)-h^{0}\left(\mathcal{O}_{Z}\right)=\chi\left(\mathcal{O}_{Z}\right)+g(Y)-1$.

Furthermore, from (1.1.7) and (1.1.4) for $m=1$,

$$
\operatorname{deg} M \leq \chi\left(\mathcal{O}_{Z}\right)+2(g(Y)-1) .
$$

On the other hand, as mentioned previously, $K_{Z}=\varphi_{\mid Z}^{*} M$. So, by (1.1.8) and the canonical bundle formula for elliptic fibrations, we conclude that $\varphi_{\mid Z}$ has no multiple fibres. This completes the proof.

Remarks 1.2. (i) As we have shown in the proof of the Theorem, $\varphi_{\mid Z}$ has no multiple fibres. Hence the equality holds in (1.1.8). This directly implies that the equalities also hold in (1.1.7) and (1.1.6). In particular, it follows from (1.1.6) that

$$
h^{0}(M)=p_{g}(Z) \quad \text { if } \quad K_{X}+\operatorname{det} \mathcal{E} \quad \text { is nef. }
$$

(ii) The morphism $\varphi: X \rightarrow Y$ considered above comes from the Stein factorization of the morphism associated with $\left|m\left(K_{X}+\operatorname{det} \mathcal{E}\right)\right|$ for $m$ large enough, when $K_{X}+\operatorname{det} \mathcal{E}$ is nef. Since $K_{Z}^{2}=0$, we see that $K_{X}+\operatorname{det} \mathcal{E}$ is not ample. At this point we could refer to a result of Andreatta and Mella listing the possible structures of $(X, \mathcal{E})$, depending on whether $K_{X}+\operatorname{det} \mathcal{E}$ is big or not [AM, Theorem 5.1, parts 2) and 3) respectively]. Checking which of these structures is compatible with condition $(*)$ and our assumption that $Z$ is a minimal surface with $\kappa(Z)=1$ leads to subcase (ii) in [AM, Theorem 5.1, part 2)]. This gives an alternate proof to our first claim that $\operatorname{dim} Y=1$, with the general fibre $F$ of $\varphi$ satisfying the condition $K_{F}+\operatorname{det} \mathcal{E}_{F}=\mathcal{O}_{F}$.

Corollary 1.3. Let $X^{\prime}$ and $\mathcal{E}^{\prime}$ be as in case (b) of the Theorem. Then

$$
h^{0}\left(m\left(K_{X^{\prime}}+\operatorname{det} \mathcal{E}^{\prime}\right)\right)=P_{m}(Z)=m\left(\chi\left(\mathcal{O}_{Z}\right)+2 g(Y)-2\right)+1-g(Y)
$$

for all $m \geq 1$.

Proof. Since $P_{m}(Z)$ and $\chi\left(\mathcal{O}_{Z}\right)$ are birational invariants, it is enough to prove the formula for $Z^{\prime}$ instead of $Z$. Since $K_{X^{\prime}}+\operatorname{det} \mathcal{E}^{\prime}$ is nef, combining (1.1.2) with (1.1.4) gives $h^{0}\left(m\left(K_{X^{\prime}}+\operatorname{det} \mathcal{E}^{\prime}\right)\right)=m \operatorname{deg} M+1-g(Y)$ for $m \geq 1$. Moreover, by Remark 1.2 (i) we know that the equality holds in (1.1.8). In other words, $\operatorname{deg} M=\chi\left(\mathcal{O}_{Z^{\prime}}\right)+2 g(Y)-2$. Therefore

$$
h^{0}\left(m\left(K_{X^{\prime}}+\operatorname{det} \mathcal{E}^{\prime}\right)\right)=m\left(\chi\left(\mathcal{O}_{Z^{\prime}}\right)+2 g(Y)-2\right)+1-g(Y)
$$

for all $m \geq 1$. Next we consider $P_{m}\left(Z^{\prime}\right)$. As we showed in $1.1, Z^{\prime}$ is a minimal surface with $\kappa\left(Z^{\prime}\right)=1$, the elliptic fibration $\varphi_{\mid Z^{\prime}}: Z^{\prime} \rightarrow Y$ has 
no multiple fibres, and $K_{Z^{\prime}}=\varphi_{\mid Z^{\prime}}^{*} M$ for some ample line bundle $M$ on $Y$. Hence, by Lemma 0.5

$$
P_{m}\left(Z^{\prime}\right)=m\left(\chi\left(\mathcal{O}_{Z^{\prime}}\right)+2 g(Y)-2\right)+1-g(Y)
$$

for every $m \geq 2$. Now assume that $M=K_{Y}$. Then $h^{1}(M)=1$. However, this contradicts (1.1.3). Thus (1.3.1) is also valid for $m=1$ by Lemma 0.5 again.

Corollary 1.4. Let $X, \mathcal{E}$ and $Z$ be as in $(*)$. Then $Z$ cannot be the product of an elliptic curve $E$ and a smooth curve $Y$ of genus $g(Y) \geq 1$.

Proof. By contradiction, assume that $Z=E \times Y$. If $g(Y)=1$, then $Z$ is an abelian surface, which cannot occur in view of [L1, Corollary 1.5]. If $g(Y) \geq 2$, then $K_{Z}=p^{*} K_{Y}$, where $p: Z \rightarrow Y$ denotes the projection, hence $Z$ is a minimal surface with $\kappa(Z)=1$ and our Theorem applies. Case (a) cannot hold, since $Z$ is minimal. On the other hand, in case (b) we have $h^{1}\left(\mathcal{O}_{Z}\right)=g(Y)$ by (1.1.1). But this gives a contradiction, since $h^{1}\left(\mathcal{O}_{E \times Y}\right)=g(E)+g(Y)=1+g(Y)$.

This generalizes a well-known fact in the setting of ample divisors $[\mathbf{S} 1$, Proposition IV]. We would like to point out that by the same argument as in the proof of Corollary 1.4 we can also exclude the case where $Z$ is an elliptic fibre bundle over a smooth curve of positive genus.

\section{Kodaira dimensions.}

Let things be as in the Introduction. In [LM1] we conjectured that if $Z$ has Kodaira dimension $\kappa(Z)<2$ then $\kappa(X)=-\infty$. The fact that $\kappa(X)=-\infty$ was proved when $\kappa(Z)=-\infty$ in [LM1, Corollary 5.2] and when $\kappa(Z)=0$ in $[\mathbf{L} 1$, Corollary 4.3]. At present our conjecture follows from a more general result of Peternell, Schneider and Sommese on the Kodaira dimension of subvarieties, obtained in connection with the theory of normal pairs $(X, A)$ with $\mathbb{Q}$-effective normal sheaf $\mathcal{N}_{A / X}$ [PSS, Theorem 4.13]. Here we apply our Theorem to give an elementary proof of the conjecture above.

Corollary 2.1. Let $X, \mathcal{E}$ and $Z$ be as in $(*)$ and assume that $\kappa(Z)<2$. Then $\kappa(X)=-\infty$.

Proof. By what we mentioned before we can assume that $\kappa(Z)=1$. Then $(X, \mathcal{E})$ is as in the Theorem. In case (a), $X$ is a $\mathbb{P}^{n-2}$ bundle over a smooth surface $S$. Hence by the easy addition theorem we get $\kappa(X) \leq \kappa\left(\mathbb{P}^{n-2}\right)+$ $\operatorname{dim} S=-\infty$. In case (b), up to the birational morphism $f$, which does not affect the Kodaira dimension, we can suppose that $X$ fibres over a smooth curve $Y$, with the general fibre being a smooth projective variety $F$ such that $K_{F}+\operatorname{det} \mathcal{E}_{F}=\mathcal{O}_{F}$. In particular $F$ is Fano and so $\kappa(F)=-\infty$. Then the easy addition theorem again gives $\kappa(X) \leq \kappa(F)+\operatorname{dim} Y=-\infty$. 
Another consequence of Section 1 is the following:

Corollary 2.2. Let $X, \mathcal{E}, Z$ be as in case (b) of the Theorem. Then $p_{g}(Z)>0$.

Proof. In view of the birational invariance of $p_{g}$ we can assume that $Z=Z^{\prime}$. Thus, we know by 1.1 that the elliptic fibration of $Z$ is given by $\varphi_{\mid Z}: Z \rightarrow Y$ with $K_{Z}=\varphi_{\mid Z}^{*} M$ for some ample $M \in \operatorname{Pic}(Y)$. In particular, $\operatorname{deg} M>0$. Moreover, by (1.2.1) we have $p_{g}(Z)=h^{0}(M)$. Since $\kappa(Z)=1$, we know by the Castelnuovo-de Franchis theorem that $\chi\left(\mathcal{O}_{Z}\right) \geq 0$. On the other hand, by (1.1.1) we have $g(Y)=h^{1,0}(Z)$. So, if $p_{g}(Z)=0$, combining this equality with the inequality above implies $1-g(Y) \geq 0$. But then, by (1.1.4) for $m=1$, we get

$$
p_{g}(Z)=h^{0}(M)=\operatorname{deg} M+1-g(Y) \geq 1,
$$

a contradiction.

Note that the same property expressed by Corollary 2.2 still holds if $Z$ is a surface of Kodaira dimension zero. Actually, in this case, if $X, \mathcal{E}$ and $Z$ are not as in case (a) (case (1) in $[\mathbf{L} 1$, Theorem]), after contracting a finite number of $(-1)$-hyperplanes we get a new triplet $\left(X^{\prime}, \mathcal{E}^{\prime}, Z^{\prime}\right)$ satisfying (*), with $K_{X^{\prime}}+\operatorname{det} \mathcal{E}^{\prime}$ nef and $Z^{\prime}$ a minimal surface with $\kappa\left(Z^{\prime}\right)=0$. In particular, $12 K_{Z^{\prime}}=\mathcal{O}_{Z^{\prime}}$. The injectivity of the restriction homomorphism $\operatorname{Pic}\left(X^{\prime}\right) \rightarrow \operatorname{Pic}\left(Z^{\prime}\right)$ [LM2, (1.1.6)] combined with the fact that $\left(K_{X^{\prime}}+\right.$ $\left.\operatorname{det} \mathcal{E}^{\prime}\right)_{Z^{\prime}}=K_{Z^{\prime}}$ implies that $12\left(K_{X^{\prime}}+\operatorname{det} \mathcal{E}^{\prime}\right)=\mathcal{O}_{X^{\prime}}$. Hence $X^{\prime}$ is Fano, but since Fano manifolds have no torsion in their Picard groups, we conclude that $K_{X^{\prime}}+\operatorname{det} \mathcal{E}^{\prime}=\mathcal{O}_{X^{\prime}}$. Hence, by adjunction, $K_{Z^{\prime}}=\mathcal{O}_{Z^{\prime}}$, which gives $p_{g}(Z)=p_{g}\left(Z^{\prime}\right)=1$.

In $[\mathbf{L 2},(4.2)]$ the first author formulated the following conjecture: Let $X$, $\mathcal{E}$ and $Z$ be as in $(*)$. If $p_{g}(Z)=0$ and $\kappa(Z) \geq 0$, then $(X, \mathcal{E})$ is as in case (a) of the Theorem. Corollary 2.2 and the discussion above show that this conjecture is true when $\kappa(Z)=0$ or 1 .

\section{References}

[AM] M. Andreatta and M. Mella, Contractions on a manifold polarized by an ample vector bundle, Trans. Amer. Math. Soc., 349 (1997), 4669-4683, MR 98b:14012, Zbl 885.14004.

[BG] S. Bloch and D. Gieseker, The positivity of the Chern classes of an ample vector bundle, Invent. Math., 12 (1971), 112-117, MR 45 \#6825, Zbl 212.53502.

[D] H. D'Souza, Threefolds whose hyperplane sections are elliptic surfaces, Pacific J. Math., 134 (1988), 57-78, MR 89i:14028, Zbl 626.14031.

[F] R. Friedman, Algebraic Surfaces and Holomorphic Vector Bundles, Springer, New York, 1998, MR 99c:14056, Zbl 902.14029. 
[KMM] Y. Kawamata, K. Matsuda and K. Matsuki, Introduction to the minimal model problem, in 'Algebraic Geometry', Sendai, 1985 (ed. T. Oda), 10, Adv. Stud. Pure Math., Kinokuniya, Tokyo, (1987), 283-360, MR 89e:14015, Zbl 672.14006.

[L1] A. Lanteri, Ample vector bundles with sections vanishing on surfaces of Kodaira dimension zero, Matematiche (Catania), 51 (1996), 115-125, MR 99a:14066, Zbl 910.14022.

[L2] - Geometric genera for ample vector bundles with regular sections, Rev. Mat. Complut., 13(1) (2000), 33-48, CMP 1794902.

[LM1] A. Lanteri and H. Maeda, Geometrically ruled surfaces as zero loci of ample vector bundles, Forum Math., 9 (1997), 1-15, MR 97i:14027, Zbl 876.14026.

[LM2] _ Ample vector bundle characterizations of projective bundles and quadric fibrations over curves, in 'Higher Dimensional Complex Varieties', Trento, 1994 (eds. M. Andreatta and T. Peternell), Walter de Gruyter, Berlin-New York, (1996), 247-259, MR 98h:14051, Zbl 891.14011.

[M] H. Maeda, Nefness of adjoint bundles for ample vector bundles, Matematiche (Catania), 50 (1995), 73-82, MR 97f:14042, Zbl 865.14024.

[PSS] T. Peternell, M. Schneider and A.J. Sommese, Kodaira dimension of subvarieties, Internat. J. Math., 10 (1999), 1065-1079, CMP 1739364.

[PSW] T. Peternell, M. Szurek and J.A. Wiśniewski, Fano manifolds and vector bundles, Math. Ann., 294 (1992), 151-165, MR 93h:14030, Zbl 786.14027.

[S1] A.J. Sommese, On manifolds that cannot be ample divisors, Math. Ann., 221 (1976), 55-72, MR 53 \#8503, Zbl 316.14006.

[S2] _ The birational theory of hyperplane sections of projective threefolds, Unpublished preprint, 1981.

[S3] - On the nonemptiness of the adjoint linear system of a hyperplane section of a threefold, J. Reine Angew. Math., 402 (1989), 211-220, MR 91h:14013, Zbl 675.14005.

Received October 21, 1999.

Dipartimento di Matematica "F. Enriques"

Università degli Studi di Milano

Via C. Saldini, 50

I-20133 Milano

ITALY

E-mail address: lanteri@mat.unimi.it

Department of Mathematical Sciences

School of SCIENCE ANd ENGINEERING

WASEDA UNIVERSITY

3-4-1 Ohkubo, Shinjuku

TOKYO 169-8555

JAPAN

E-mail address: hmaeda@mse.waseda.ac.jp 